
\title{
R Research S Surare \\ USP24-GSDMB complex promotes bladder cancer proliferation via activation of the STAT3 pathway
}

\section{Haiqing He}

Second Xiangya Hospital

\section{Bin Zhang}

Second Xiangya Hospital

\section{Bin Yan}

Second Xiangya Hospital

Ming Xiao

Second Xiangya Hospital

Jiannan Ren

Second Xiangya Hospital

\section{Dong Zi}

Second Xiangya Hospital

\section{Liang Zhu}

Second Xiangya Hospital

\section{Zhaohui Zhong}

Second Xiangya Hospital

\section{Xiaokun Zhao}

Second Xiangya Hospital

xin Jin ( $\square$ dadajin888@163.com )

Second Xiangya Hospital https://orcid.org/0000-0001-7513-0318

\section{Wei Xiong}

Second Xiangya Hospital

\section{Research}

Keywords: GSDMB, STAT3, USP24, bladder cancer

Posted Date: October 27th, 2020

DOI: https://doi.org/10.21203/rs.3.rs-95101/v1

License: (c) (1) This work is licensed under a Creative Commons Attribution 4.0 International License. Read Full License 
Version of Record: A version of this preprint was published at International Journal of Biological Sciences on January 1st, 2021. See the published version at https://doi.org/10.7150/ijbs.54442. 


\section{Abstract}

\section{Background}

Bladder cancer is the fourth and tenth most common malignancy in men and women worldwide, respectively. One of the main reasons for the unsatisfactory therapeutic control of bladder cancer is that the molecular biological mechanism of bladder cancer is complex. Gasdermin B (GSDMB) is one member of the gasdermin family and participates in the regulation of cell pyroptosis. The role of GSDMB in bladder cancer has not been studied to date.

\section{Methods}

The clinical relevance of GSDMB was examined by the TCGA data set. Functional assays, such as the MTT assay, Celigo fluorescent cell-counting assay, Annexin V-APC assay and xenografts, were used to determine the biological role of GSDMB in bladder cancer. The interaction between GSDMB and STAT3, or GSDMB and USP24 were detected by Mass spectrometry and verified through immunoprecipitation. The relationship between USP24, GSDMB and STAT3 was examined by Western blot analysis and immunohistochemistry.

\section{Results}

In this study, bioinformatics analysis indicated that the mRNA expression level of GSDMB in bladder cancer tissues was higher than that in adjacent normal tissues. Then, we showed that GSDMB promoted bladder cancer progression. Furthermore, we demonstrated that GSDMB interacted with STAT3 to increase the phosphorylation of STAT3 and modulate the glucose metabolism and promote tumor growth in bladder cancer cells. Besides, we also showed that USP24 stabilized GSDMB to activate STAT3 signaling, which was blocked by the USP24 inhibitor.

\section{Conclusions}

We suggested that aberrantly up-regulated GSDMB was responsible for enhancing the growth and invasion ability of bladder cancer cells. Then, we showed that GSDMB could bind to STAT3 and activate STAT3 signaling in bladder cancer. Furthermore, we also demonstrated that USP24 interacts with GSDMB and prevents GSDMB from degradation in bladder cancer cells. Therefore, the USP24/GSDMB/STAT3 axis may be a new targetable signaling pathway for bladder cancer treatment.

\section{Background}

Bladder cancer is the fourth and tenth most common malignancy in men and women worldwide, respectively ${ }^{1}$. According to the pathological characteristics of patients with bladder cancer, clinicians choose surgical treatment, radiotherapy, chemotherapy, immunotherapy, bladder perfusion therapy, and comprehensive treatment for different stages of bladder cancer. However, each type of cancer exhibits specific problems: for non-muscle invasive bladder cancer, a high postoperative recurrence rate is the 
main clinical problem, while for muscle invasive bladder cancer, the prognosis is very poor, and only few patients survived more than 5 years ${ }^{2,3}$. One of the main reasons for the unsatisfactory therapeutic control of bladder cancer is that the molecular biological mechanism of bladder cancer is complex. Therefore, it is of great significance to identify the driving factors and inhibitors of bladder cancer and understand its mechanism of action for finding new therapeutic targets of bladder cancer.

The Janus kinases (JAK)/signal transducer and activator of transcription 3 (STAT3) axis is abnormally activated in bladder cancer and correlated with the poor prognosis ${ }^{4-6}$. STAT3 regulates the genes associated with promoting cancer cell proliferation, invasion, and metastasis, as well as changes in metabolism ${ }^{7,8}$. Furthermore, STAT3 plays an important role in modulating the inflammation and immune response of the tumor microenvironment ${ }^{9,10}$. Thus, STAT3 is considered to be an ideal target for bladder cancer therapy. In addition to the classical IL6/JAK/STAT3 pathway ${ }^{11}$, other pathways participate in the activation of STAT3 in bladder cancer. For instance, reactive oxygen species and glutaminolysis are reported to activate STAT3 in bladder cancer ${ }^{8}$. Moreover, the PD-L1/ITGA6 axis promotes STAT3 nucleus translocation in bladder cancer ${ }^{10}$. Furthermore, several proteins (e.g., EZH2 ${ }^{12}$ or Msi2 ${ }^{13}$ ), long noncoding RNA (e.g., SNHG16 ${ }^{14}$ ), and micro RNA (e.g., miR-98 ${ }^{14}$ or miR-4324) have been reported to be responsible for STAT3 activation in bladder cancer cells. Therefore, further exploring the regulatory mechanism of STAT3 in cells could provide therapeutic strategies for bladder cancer.

Gasdermin B (GSDMB) is one member of the gasdermin family and participates in the regulation of cell pyroptosis ${ }^{15}$. The role of GSDMB in bladder cancer has not been studied to date. In this study, we systematically studied the clinical pathological and biological effects of GSDMB in bladder cancer. We found that GSDMB bound to and activated STAT3 to modulate the glucose metabolism and promote tumor growth in bladder cancer cells. Furthermore, we showed that USP24 could stabilize GSDMB, and the USP24/GSDMB/STAT3 signaling axis provided some potential therapeutic targets for bladder cancer.

\section{Material And Methods}

\section{Cell lines}

The bladder cancer cell lines 5637 and T24 were obtained from the Chinese Academy of Science Cell Bank (Shanghai, China). These cells were cultured in Dulbecco's modified Eagle's medium (DMEM; Thermo Fisher Scientific, China) with 10\% fetal bovine serum (FBS; Thermo Fisher Scientific, China) and maintained in a $37^{\circ} \mathrm{C}$ incubator supplied with $5 \% \mathrm{CO}_{2}$.

\section{Transfection and reagents}

The cells were transfected with the indicated plasmids or shRNAs with Lipofectamine 2000 (Thermo Fisher Scientific, China) according to the manufacturer's instructions. The plasmids of GSDMB and USP24 were purchased from Viogene Bioscience (China) and GENECHEM (China), respectively. The 
shRNAs were obtained from Sigma-Aldrich (USA). The chemical reagents cryptotanshinone (Cat. No. S2285), EOAI3402143 (Cat. No. S6877), and MG132 (Cat. No. S2619) were purchased from Selleckchem.

\section{Immunoprecipitation and Western blot}

For immunoprecipitation, bladder cancer cells harvested from 10-cm dishes were lysed in RIPA buffer on ice for $30 \mathrm{~min}$. The supernatant was collected after centrifugation at $12000 \mathrm{~g}$ and co-culture with protein $\mathrm{A} / \mathrm{G}$ beads and IgG antibody or primary antibody at $4{ }^{\circ} \mathrm{C}$ overnight. The beads were washed with RIPA buffer for 6 times. Then, $60 \mu \mathrm{L} 1 \mathrm{X}$ loading buffer was added to the beads, followed by boiling in hot water $\left(100^{\circ} \mathrm{C}\right)$ for $10 \mathrm{~min}$.

For the Western blot assay, the cells were lysed in RIPA buffer, and the supernatant was collected after centrifugation at $12000 \mathrm{~g}$. Then, $4 \mathrm{X}$ loading buffer was added to the supernatant, followed by boiling in hot water $\left(100^{\circ} \mathrm{C}\right)$ for $10 \mathrm{~min}$. Then, the supernatant was subjected to electrophoresis on SDS-PAGE gels. The proteins were transferred to a PVDF membrane and incubated with the primary antibody overnight at $4{ }^{\circ} \mathrm{C}$. On the next day, the membrane was washed with RIPA buffer and incubated with the secondary antibody for $2 \mathrm{~h}$ at room temperature. The chemiluminescence was measured using the Chemiluminescence Western Blot Detection Kit (Cat. No. 32209, Thermo Fisher Scientific, USA). The following primary antibodies were used for Western blotting: USP24 (Proteintech, 13126-1-AP; 1:1000 dilution), GSDMB (Proteintech, 12885-1-AP; 1:1000 dilution), GAPDH (ab8245, Abcam; 1:5000 dilution), STAT3 (Cell Signaling Technology, 30835S; 1:2000 dilution), and p-STAT3 Y705 (Cell Signaling Technology, 9145S; 1:1000 dilution). Protein levels were evaluated using the ImageJ software (National Institutes of Health).

\section{Glucose consumption and lactate production assay}

The bladder cancer cells were infected with the indicated constructs for $72 \mathrm{~h}$. The spent medium was exchanged by fresh medium, and the cells were cultured for another $24 \mathrm{~h}$. Then, the medium was collected for Glucose consumption analysis and lactate production assay. The glucose (GO) assay kit (Cat. No. GAGO20, Sigma-Aldrich, USA) was used for measuring the glucose level following the manufacturer's instructions. The lactate assay kit (Cat. No. MAK064, Sigma-Aldrich, USA) was applied to detect the lactate level according to the manufacturer's instruction.

\section{In vitro cell proliferation assay}

For the MTT assay, an equal number of bladder cancer cells were plated in a 96-well plate and maintained in an incubator at $37^{\circ} \mathrm{C}$ in $5 \% \mathrm{CO}_{2}$ atmosphere. Then, $20 \mu \mathrm{L}$ of MTT $(5 \mathrm{mg} / \mathrm{mL}$, Genview, Cat. No. JT343) was added to each well followed by incubation for $4 \mathrm{~h}$ at $37^{\circ} \mathrm{C}$. Then, the supernatants were removed, and $100 \mu \mathrm{L}$ DMSO were added to the supernatants. The absorbance at $490 \mathrm{~nm}$ was measured to determine the cell proliferation rate. 
For the Celigo fluorescent cell-counting assay, an equal number of bladder cancer cells were plated in a 96-well plate and maintained in an incubator at $37^{\circ} \mathrm{C}$ in $5 \% \mathrm{CO}_{2}$ atmosphere. The number of cells was measured using the Celigo machine (Nexcelom).

\section{In vivo cell growth assay}

All animal experiments were approved by the Ethics Committee of Xiangya Medical College, Central South University. BALB/c-nude mice ( 6 weeks old) were purchased from Vitalriver (Beijing, China). T24 bladder cancer cells were transfected with the indicated shRNAs. After $48 \mathrm{~h}$, the cells $\left(2 \times 10^{7}\right.$ per mouse) were subcutaneously injected into the left back of the mice. The length and width of tumors were measured using a Vernier caliper, and tumor volumes were calculated using the formula $\left(L \times W^{2}\right) / 2$. After the mice were euthanized, tumors were excised and weighted.

\section{Quantitative real-time PCR and chromatin immunoprecipitation ChIP-qPCR}

The TRIzol reagent (Thermo Fisher Scientific, USA) was used to extract the total RNA from the cells. Using the Reverse Transcription kit (PrimeScript ${ }^{\text {TM }}$ RT reagent Kit, Code No. RR037A, Takara Bio Inc. Shigo, Japan), cDNA was synthesized from $1 \mu \mathrm{g}$ RNA, and real-time PCR analysis was carried out with a PCR kit (TB Green ${ }^{\text {TM }}$ Fast qPCR Mix, Code No. RR430A, Takara Bio Inc. Shigo, Japan) according to the manufacturer's instructions. All obtained values were normalized to that of GAPDH, and the $2^{-\triangle \triangle C t}$ method was used to quantify fold changes. ChIP was performed with Chromatin Extraction Kit (Abcam, ab117152, USA) and ChIP Kit Magnetic - One Step (Abcam, ab156907, USA) following the manufacturer's instructions. STAT3 (Cell signaling Technology, 30835S; 1:500) was used for the ChIP assay. Purified DNA was analyzed by real-time PCR using a PCR kit (Takara Bio Inc., Japan) according to the manufacturer's protocol.

\section{Tissue microarray and immunohistochemistry}

The tissue microarray (Cat No. DC-Bla 1002a, Avilabio, Xian, China) was employed to evaluated the relationship between USP24 and GSDMB. The following antibodies were used for immunohistochemistry: USP24 (Proteintech, 13126-1-AP; 1:400) and GSDMB (Proteintech, 12885-1-AP; 1:800). The immunohistochemistry score equals to the staining intensity score multiplied by the proportion of positive tumor cells. The staining intensity was graded as follows: $1=$ weak staining at $100 \times$ magnification but little or no staining at $40 \times$ magnification; $2=$ medium staining at $40 \times$ magnification; 3 $=$ strong staining at $40 \times$ magnification. The immunostaining intensity was evaluated for scoring independently by two experienced pathologists who were blinded for the detailed information.

\section{Statistical analysis}

All data are presented as the mean \pm standard deviation (SD). Statistical significance was determined by one- or two-way ANOVA using the GraphPad Prism 5 software. P-values $<0.05$ were considered statistically significant. 


\section{Results}

\section{GSDMB promotes bladder cancer progression in vitro and in vivo}

First, we explored the clinical biological effects of GSDMB in bladder cancer. It is worth noting that the mRNA expression level of GSDMB in bladder cancer tissues was higher than that in adjacent normal tissues according to the bioinformatics analysis of the TCGA data set $(n=19, P=1.98 \mathrm{e}-2$; Fig. $1 A-C)$. To further study the biological role of GSDMB in bladder cancer, GSDMB was knocked down by two different short harpin RNAs (shRNAs) in both T24 and 5637 cell lines (Fig. 1D). In vitro cell proliferation assays showed that the growth activity of bladder cancer cells was significantly inhibited after GSDMB was knocked down (Fig. 1E-G). We also found that the apoptotic rate of bladder cancer cells in the GSDMB silencing group was significantly higher than that in the control group in both 5637 and T24 cancer cells, as analyzed by the Annexin V-APC assay (Fig. 1H). The cell cycle analysis also showed that the number of bladder cancer cells in the S phase decreased when GSDMB was knocked down (Supplementary Fig. $1 \mathrm{~A})$, indicating slow cell division and proliferation. In addition to the in vitro experiments, we also employed the nude mice xenograft tumor model after inhibition of the expression of GSDMB to study the growth-promoting effect of GSDMB in bladder cancer in vivo. The results revealed significantly reduced tumor volume and mass and even no tumor after knocking down GSDMB, which was consistent with the results of the in vitro experiments (Fig. 1I-K). Furthermore, the invasion and migration ability of the cells was also significantly weakened after knocking down GSDMB (Supplementary Fig. 1B). Concluding, GSDMB promotes bladder cancer progression in cells and in mice.

\section{GSDMB regulates the glycolysis of bladder cancer cells}

Given the critical role of GSDMB in bladder cancer, we investigated the mechanism by which GSDMB promotes the progression of bladder cancer. We conducted RNA-Seq analysis after using small interfering RNA (siRNA) to inhibit the expression of GSDMB (Fig. 2A, B). Then, we performed GO and KEGG enrichment analysis, which indicated that GSDMB had a significant influence on glycolysis and gluconeogenesis (Fig. 2C, D). To further confirm this mechanism, we knocked down or overexpressed GSDMB in T24 cells (Fig. 2E, I, respectively). The mRNA expression levels of the genes HK2, LDHA, and ENO2, which are related to the glucose metabolism pathway, were markedly decreased when GSDMB was knocked down (Fig. 2F). Moreover, GSDMB knock down conveniently reduced glucose consumption and lactate production corresponding to the amount of GSDMB knock down (Fig. 2G, H). In contrast, overexpression of GSDMB had the inverse effect (Fig. 2J-L). Collectively, our data suggest that GSDMB can regulate the glycolysis of bladder cancer cells.

\section{GSDMB regulates the glycolysis via activating STAT3 in bladder cancer cells}

In the following, the mechanism underlying the regulation of the glycolysis of bladder cancer cells by GSDMB was studied. We first performed mass spectrometry to identify the potential binding partners of GSDMB (Fig. 3A and Table S1), revealing that GSDMB may bind to STAT3. This finding was confirmed by immunoprecipitation in both T24 and 5637 bladder cancer cells (Fig. 3B). Multiple studies showed that 
STAT3 exerts a significant effect on the glycolysis and glucose-dependent addiction of tumor cells, which is known as the Warburg effect ${ }^{16,17}$. Specifically, it has been reported that STAT3 regulates the glucose metabolism through increasing the HK2 expression in cancer cells ${ }^{18}$. Furthermore, STAT3 up-regulates LDHA expression to promote the proliferation of urinary bladder cancer cells ${ }^{19}$. Interestingly, GSDMB and STAT3 proteins could precipitate each other (Fig. 3A, B). Moreover, GSDMB modulates the glycolysis by up-regulating the expression of HK2 in bladder cancer cells (Fig. 2E-2L), suggesting that GSDMB may be involved in regulating the glucose metabolism of bladder cancer cells through STAT3-related signaling pathways. To further verify that GSDMB regulates the glucose metabolism via the STAT3 pathway, we suppressed the expression of STAT3 while GSDMB was knocked down or overexpressed. The results showed that the protein and mRNA levels of HK2 remained constant after knocking down STAT3 whether GSDMB was inhibited or overexpressed (Fig. 3C-F), indicating that STAT3 is an important intermediate mediator for GSDMB in regulating the glucose metabolism. Furthermore, it has been reported that Tyr 705 STAT3 phosphorylation leads to downstream glucose metabolism disorders ${ }^{20,21}$. Importantly, the protein level of p-STAT3 Tyr705 was down-regulated when GSDMB was knocked down (Fig. 3G). On the contrary, p-STAT3 Tyr705 increased as the overexpression level of GSDMB increased (Fig. 3H). However, the total STAT3 level did not change whether GSDMB was knocked down or overexpressed. Furthermore, we used cryptotanshinone to inhibit the phosphorylation of Tyr 705 STAT3 in bladder cancer cells with knocked down or overexpressed GSDMB, resulting in constant protein and mRNA levels of HK2 (Fig. 3I-L). These data suggested that GSDMB regulates the glucose metabolism via promoting the phosphorylation of Tyr 705 STAT3 in bladder cancer cells.

\section{GSDMB-STAT3 signaling regulates IGFBP3 expression in bladder cancer}

The RNA-Seq assay revealed 27 up-regulated genes and 110 down-regulated genes with IGFBP3 as the most significantly down-regulated gene after silencing GSDMB in T24 cells. To verify the accuracy of these results, we knocked down or overexpressed GSDMB and found a positive correlation with the change in the protein and mRNA expression of IGFBP3 in both T24 and 5637 cells (Fig. 4A-D). IGFBP3 is closely related to the glucose metabolism in cells (Saukkonen et al., 2006; Wang et al., 2013). The above data indicated that GSDMB may activate STAT3 to regulate the glycolysis in bladder cancer cells.

Therefore, we speculated that GSDMB increased IGFBP3 in a STAT3-dependent manner. Notably, the knockdown of STAT3 inhibited the expression of IGFBP3 at the transcription and translation levels (Fig. 4E, F). Furthermore, we found that STAT3 could bind to the promoter of IGFBP3 (Fig. 4G, H), indicating that STAT3 acted as a transcription factor to initiate the transcription of IGFBP3 in bladder cancer cells. Furthermore, we knocked down the expression of STAT3 while GSDMB was knocked down or overexpressed, and the results showed that IGFBP3 remained constant (Fig. 4I-L), which confirmed our speculation. In conclusion, GSDMB regulates IGFBP3 expression through the STAT3 pathway in bladder cancer.

Moreover, the rate of cell proliferation did not significantly change after the simultaneous knock down of GSDMB and STAT3, while it decreased after STAT3 knock down alone (Fig. 4M, N). The results of the in vivo experiments were also completely consistent with these results, and no statistical difference in the 
tumor mass or volume was detected between the two groups, which further demonstrated that GSDMB promoted the progression of bladder cancer by regulating STAT3.

\section{USP24 interacts with GSDMB to up-regulate the GSDMB protein level in bladder cancer}

After revealing the important role of GSDMB in bladder cancer, we studied how GSDMB was regulated in bladder cancer for the targeted treatment of bladder cancer. Mass spectrometry of GSDMB indicated that GSDMB may interact with USP24 (Table S1), which was verified in bladder cancer cells through immunoprecipitation (Fig. 5A). Interestingly, GEPIA network tool analysis showed that there was no correlation between GSDMB and USP24 in their RNA levels (Fig. 5B). To analyze the protein expression levels of GSDMB and USP24, a bladder cancer tissue microarray $(n=80)$ was performed, and obtained typical images are displayed in Fig. 5C. The results showed a significant positive correlation between GSDMB and USP24 proteins in clinical specimens (Fig. 5D). As a deubiquitinating enzyme, ubiquitinspecific peptidase USP24 could deubiquitinate downstream proteins to maintain their stability 22,23 . Thus, USP24 may mediate the deubiquitination of GSDMB and maintain its stability. Consistently, the protein expression level of GSDMB also decreased or increased after knock down or overexpression of USP24, respectively, while the mRNA level of GSDMB remained constant (Fig. 5E-H). Furthermore, the changes in GSDMB observed after treatment with USP24 inhibitor EOAI3402143 were exactly the same as those observed after knocking down USP24 (Fig. 5I, J). To sum up, USP24 interacts with GSDMB to up-regulate the GSDMB protein level in bladder cancer.

\section{USP24 stabilizes GSDMB to promote STAT3 phosphorylation in bladder cancer cells}

Given that USP24 promoted the expression of the GSDMB protein levels rather than that of the mRNA levels in bladder cancer cells, we hypothesized that USP24 may regulate the stability of GSDMB through the ubiquitinated proteasome pathway. We have shown that knocking down USP24 using shUSP24 or USP24 inhibitor EOAI3402143 could reduce the protein expression of GSDMB, but the process was inhibited by proteasome inhibitor MG132 (Fig. 6A, B, E, F). Moreover, the protein half-life of GSDMB was significantly decreased when USP24 was knocked out or inhibited, while overexpression of USP24 showed the opposite effect (Fig. 6C, G). The proteasome recognized and degraded ubiquitinated proteins ${ }^{24}$. We proved that knocking down or inhibiting USP24 increased the polyubiquitination of GSDMB, but overexpressing USP24 decreased the polyubiquitination of GSDMB in bladder cancer cells (Fig. 6D, H). These data suggest that USP24 promoted the stability of the GSDMB protein in bladder cancer by deubiquitinating GSDMB.

Moreover, after USP24 was inhibited or overexpressed, the expression level of p-STAT3 Y705 also decreased or increased, respectively (Fig. 6I-K). This raised the question if USP24 regulates the activation of the STAT3 signaling pathway through GSDMB. To verify this hypothesis, we knocked down the expression of GSDMB while knocking down or suppressing the expression of USP24. The results showed that the p-STAT3 Y705 protein level remained stable, while it decreased when GSDMB was knocked down alone (Fig. 6I, J). Similarly, overexpressing USP24 after knocking down GSDMB also 
resulted in a constant p-STAT3 Y705 protein level (Fig. 6K). Therefore, the USP24-GSDMB pathway axis played a key role in modulating the phosphorylation of STAT3. In conclusion, USP24 stabilizes GSDMB to promote STAT3 phosphorylation in bladder cancer cells (Fig. 6L).

\section{Discussion}

Gasdermin (GSDM) was first found in the epithelial tissues of the gastrointestinal tract and skin and consists of the six members GSDMA, GSDMB, GSDMC, GSDMD, GSDME, and DFNB59 25, 26. GSDM gene family members have about $45 \%$ sequence homology and two domains that can bind to each other 26 . The N-terminal domain can oligomerize in the cell membrane and form a pyrolytic hole with 10-16 nm in diameter ${ }^{27}$. Smaller intracellular substances, such as interleukin (IL)-1 $\beta$ and IL-18, can be secreted through this hole. Increased pyrolytic pores eventually lead to cell scorch and release the whole cell substance ${ }^{27}$. As one member of the GSDM family, GSDMB is cleaved by caspase-1, and the release of the $\mathrm{N}$-terminal domain induces cell death ${ }^{15}$. So far, the role of GSDMB in tumors is still controversial. Some studies showed that GSDMB can promote pyroptotic death and inhibit the growth of tumor cells ${ }^{15}$. In contrast, in HER2-positive breast cancer patients, the clinical outcome of patients with high expression of GSDMB (about $60 \%$ of patients) is significantly worse, and the neoadjuvant effect is poor, which is often accompanied by trastuzumab resistance ${ }^{28,29}$. Delivery of nano antibodies against GADMB have shown an obvious inhibitory effect on breast cancer in in vivo and in vitro levels ${ }^{30}$. At present, the specific role and underlying mechanism of GSDMB in bladder cancer needs to be further explored. In this study, we showed that GSDMB was up-regulated in bladder cancer tissues compared with adjacent normal tissue. Overexpressed GSDMB promoted cancer cell growth via interacting with STAT3 to elevate the phosphorylation level of STAT3, which increased the expression of HK2, LDNA, ENO2, and IGFBP3 to enhance the glycolysis of bladder cancer cells. However, the mechanism underlying the modulation of the phosphorylation of STAT3 by GSDMB is still not fully understood, so further experimental studies are needed.

Ubiquitin-specific peptidase 24 (USP24), containing 2620 amino acids, acted as a deubiquitinase to regulate the ubiquitin modification of the substrates ${ }^{31}$. The biological role of USP24 in cancer is poorly understood. It has been reported that USP24 is overexpressed in the late stage of lung cancer ${ }^{32}$. Upregulated USP24 increased the protein level of $\beta$-TrCP and p300 to promote lung cancer malignancy ${ }^{31}$. Moreover, targeting USP24 by small inhibitors hinders the progression of T-cell acute lymphoblastic leukemia and B-cell malignancies ${ }^{33,34}$. However, some groups also demonstrated that USP24 prevents P53 from degradation to regulate the DNA damage response ${ }^{22}$. Intriguingly, USP24 up-regulates the expression of MDM2, the well-known E3 ligase of P53, in lung cancer, which contradicts the above finding ${ }^{31}$. Thus, the role of USP24 in cancer needs to be further elucidated. Here, our results indicated that USP24 bound to GSDMB to stabilize GSDMB, and subsequently activated the STAT3 pathway in bladder cancer cells. We further showed that USP24 inhibitors could block this process via inducing GSDMB degradation in cancer cells, which provided a therapeutic strategy for inhibiting the GSDMB/STAT3 axis 
in bladder cancer. In contrast, it has been reported that EOAI3402143, which we used as a USP24 inhibitor, not only inhibits the activity of USP24 but also targets USP9x and USP5 in cells ${ }^{34}$. Although we genetically silenced USP24 to prove the stabilization of GSDMB by USP24, we could not rule out that USP9x or USP5 acted as deubiquitinases of GSDMB in bladder cancer.

\section{Abbreviations}

GSDMB: Gasdermin B; STAT3: signal transducer and activator of transcription 3; JAK: Janus kinases; USP24: Ubiquitin-specific peptidase 24; ChIP: Chromatin immunoprecipitation; IHC: immunohistochemistry; MTT: 3-(45)-dimethylthiahiazo (-z-y1)-35-di- phenytetrazoliumromide

\section{Declarations}

\section{Ethical Approval and Consent to participate}

The study was conducted in accordance with the principles of the Declaration of Helsinki principles. All animal experiments were approved by the Ethics Committee of Xiangya Medical College, Central South University.

\section{Consent for publication}

All subjects have written informed consent.

\section{Availability of supporting data}

The datasets used and/or analyzed during the current study are available from the corresponding author (Wei Xiong, xiongwei001@csu.edu.cn; Xin Jin, dadajin888@163.com) on reasonable request.

\section{Competing interests}

The authors declare that they have no competing interests.

\section{Funding}

The research was supported by basic research Foundation from the Second Xiangya Hospital and Fellowship from Second Xiangya Hospital and Central South University

\section{Authors' contributions}

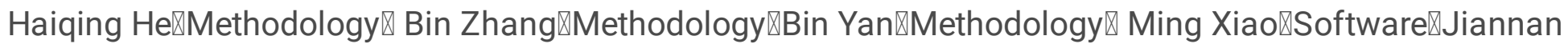

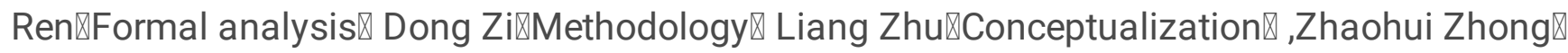

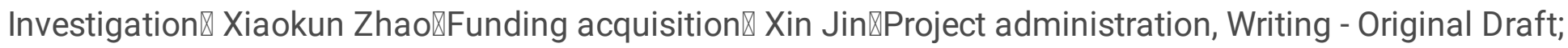
Wei Xiong $\mathbb{X}$ Funding acquisition, Project administration, Writing - Review \& Editing. 


\section{Acknowledgements}

Not applicable.

\section{References}

1. Antoni, S.; Ferlay, J.; Soerjomataram, I.; Znaor, A.; Jemal, A.; Bray, F., Bladder Cancer Incidence and Mortality: A Global Overview and Recent Trends. Eur Urol 2017,71 (1), 96-108.

2. Chou, R.; Selph, S. S.; Buckley, D. I.; Gustafson, K. S.; Griffin, J. C.; Grusing, S. E.; Gore, J. L., Treatment of muscle-invasive bladder cancer: A systematic review. Cancer 2016,122 (6), 842-51.

3. Ghandour, R.; Singla, N.; Lotan, Y., Treatment Options and Outcomes in Nonmetastatic Muscle Invasive Bladder Cancer. Trends Cancer 2019,5 (7), 426-439.

4. Santoni, M.; Conti, A.; Piva, F.; Massari, F.; Ciccarese, C.; Burattini, L.; Cheng, L.; Lopez-Beltran, A.; Scarpelli, M.; Santini, D.; Tortora, G.; Cascinu, S.; Montironi, R., Role of STAT3 pathway in genitourinary tumors. Future Sci OA 2015,1 (3), FSO15.

5. Chen, Z.; Chen, X.; Xie, R.; Huang, M.; Dong, W.; Han, J.; Zhang, J.; Zhou, Q.; Li, H.; Huang, J.; Lin, T., DANCR Promotes Metastasis and Proliferation in Bladder Cancer Cells by Enhancing IL-11-STAT3 Signaling and CCND1 Expression. Mol Ther 2019,27 (2), 326-341.

6. Goulet, C. R.; Champagne, A.; Bernard, G.; Vandal, D.; Chabaud, S.; Pouliot, F.; Bolduc, S., Cancerassociated fibroblasts induce epithelial-mesenchymal transition of bladder cancer cells through paracrine IL-6 signalling. BMC Cancer 2019,19 (1), 137.

7. Yu, H.; Lee, H.; Herrmann, A.; Buettner, R.; Jove, R., Revisiting STAT3 signalling in cancer: new and unexpected biological functions. Nat Rev Cancer 2014,14 (11), 736-46.

8. Sun, N.; Liang, Y.; Chen, Y.; Wang, L.; Li, D.; Liang, Z.; Sun, L.; Wang, Y.; Niu, H., Glutamine affects T24 bladder cancer cell proliferation by activating STAT3 through ROS and glutaminolysis. Int J Mol Med 2019,44 (6), 2189-2200.

9. Yu, H.; Pardoll, D.; Jove, R., STATs in cancer inflammation and immunity: a leading role for STAT3. Nat Rev Cancer 2009,9 (11), 798-809.

10. Cao, D.; Qi, Z.; Pang, Y.; Li, H.; Xie, H.; Wu, J.; Huang, Y.; Zhu, Y.; Shen, Y.; Zhu, Y.; Dai, B.; Hu, X.; Ye, D.; Wang, Z., Retinoic Acid-Related Orphan Receptor C Regulates Proliferation, Glycolysis, and Chemoresistance via the PD-L1/ITGB6/STAT3 Signaling Axis in Bladder Cancer. Cancer Res 2019,79 (10), 2604-2618.

11. Johnson, D. E.; O'Keefe, R. A.; Grandis, J. R., Targeting the IL-6/JAK/STAT3 signalling axis in cancer. Nat Rev Clin Oncol 2018,15(4), 234-248.

12. Chen, Z.; Du, Y.; Liu, X.; Chen, H.; Weng, X.; Guo, J.; Wang, M.; Wang, X.; Wang, L., EZH2 inhibition suppresses bladder cancer cell growth and metastasis via the JAK2/STAT3 signaling pathway. Oncol Lett 2019,18 (1), 907-915. 
13. Yang, C.; Zhang, W.; Wang, L.; Kazobinka, G.; Han, X.; Li, B.; Hou, T., Musashi-2 promotes migration and invasion in bladder cancer via activation of the JAK2/STAT3 pathway. Lab Invest 2016,96 (9), 950-8.

14. Feng, F.; Chen, A.; Huang, J.; Xia, Q.; Chen, Y.; Jin, X., Long noncoding RNA SNHG16 contributes to the development of bladder cancer via regulating miR-98/STAT3/Wnt/beta-catenin pathway axis. J Cell Biochem 2018,119 (11), 9408-9418.

15. Li, L.; Li, Y.; Bai, Y., Role of GSDMB in Pyroptosis and Cancer. Cancer Manag Res 2020,12, 3033-3043.

16. Warburg, O., On respiratory impairment in cancer cells. Science (New York, N.Y.) 1956,124 (3215), 269-70.

17. Warburg, O., On the origin of cancer cells. Science (New York, N.Y.) 1956,123 (3191), 309-14.

18. Ou, B.; Sun, H.; Zhao, J.; Xu, Z.; Liu, Y.; Feng, H.; Peng, Z., Polo-like kinase 3 inhibits glucose metabolism in colorectal cancer by targeting HSP90/STAT3/HK2 signaling. J Exp Clin Cancer Res 2019,38 (1), 426.

19. Cheng, H.; Hao, Y.; Gao, Y.; He, Y.; Luo, C.; Sun, W.; Yuan, M.; Wu, X., PLCepsilon promotes urinary bladder cancer cells proliferation through STAT3/LDHA pathwaymediated glycolysis. Oncol Rep 2019,41 (5), 2844-2854.

20. Bravard, A.; Vial, G.; Chauvin, M. A.; Rouillé, Y.; Bailleul, B.; Vidal, H.; Rieusset, J., FTO contributes to hepatic metabolism regulation through regulation of leptin action and STAT3 signalling in liver. Cell communication and signaling : CCS 2014,12, 4.

21. Dong, T.; Yan, Y.; Chai, H.; Chen, S.; Xiong, X.; Sun, D.; Yu, Y.; Deng, L.; Cheng, F., Pyruvate kinase M2 affects liver cancer cell behavior through up-regulation of HIF-1 $\mathrm{a}$ and $\mathrm{Bcl}-\mathrm{xL}$ in culture. Biomedicine \& pharmacotherapy = Biomedecine \& pharmacotherapie 2015,69, 277-84.

22. Zhang, L.; Nemzow, L.; Chen, H.; Lubin, A.; Rong, X.; Sun, Z.; Harris, T. K.; Gong, F., The deubiquitinating enzyme USP24 is a regulator of the UV damage response. Cell Rep 2015,10 (2), 140-7.

23. Wang, Y. C.; Wu, Y. S.; Hung, C. Y.; Wang, S. A.; Young, M. J.; Hsu, T. I.; Hung, J. J., USP24 induces IL-6 in tumor-associated microenvironment by stabilizing $\mathrm{p} 300$ and $\beta$-TrCP and promotes cancer malignancy. Nature communications 2018,9 (1), 3996.

24. Leestemaker, Y.; Ovaa, H., Tools to investigate the ubiquitin proteasome system. Drug Discov Today Technol 2017,26, 25-31.

25. Jorgensen, I.; Rayamajhi, M.; Miao, E. A., Programmed cell death as a defence against infection. Nat Rev Immunol 2017,17(3), 151-164.

26. Shi, J.; Gao, W.; Shao, F., Pyroptosis: Gasdermin-Mediated Programmed Necrotic Cell Death. Trends Biochem Sci 2017,42 (4), 245-254.

27. Feng, S.; Fox, D.; Man, S. M., Mechanisms of Gasdermin Family Members in Inflammasome Signaling and Cell Death. J Mol Biol 2018,430 (18 Pt B), 3068-3080.

28. Hergueta-Redondo, M.; Sarrio, D.; Molina-Crespo, A.; Megias, D.; Mota, A.; Rojo-Sebastian, A.; GarciaSanz, P.; Morales, S.; Abril, S.; Cano, A.; Peinado, H.; Moreno-Bueno, G., Gasdermin-B promotes 
invasion and metastasis in breast cancer cells. PLoS One 2014,9 (3), e90099.

29. Hergueta-Redondo, M.; Sarrio, D.; Molina-Crespo, A.; Vicario, R.; Bernado-Morales, C.; Martinez, L.; Rojo-Sebastian, A.; Serra-Musach, J.; Mota, A.; Martinez-Ramirez, A.; Castilla, M. A.; Gonzalez-Martin, A.; Pernas, S.; Cano, A.; Cortes, J.; Nuciforo, P. G.; Peg, V.; Palacios, J.; Pujana, M. A.; Arribas, J.; Moreno-Bueno, G., Gasdermin B expression predicts poor clinical outcome in HER2-positive breast cancer. Oncotarget 2016,7 (35), 56295-56308.

30. Molina-Crespo, A.; Cadete, A.; Sarrio, D.; Gamez-Chiachio, M.; Martinez, L.; Chao, K.; Olivera, A.; Gonella, A.; Diaz, E.; Palacios, J.; Dhal, P. K.; Besev, M.; Rodriguez-Serrano, M.; Garcia Bermejo, M. L.; Trivino, J. C.; Cano, A.; Garcia-Fuentes, M.; Herzberg, O.; Torres, D.; Alonso, M. J.; Moreno-Bueno, G., Intracellular Delivery of an Antibody Targeting Gasdermin-B Reduces HER2 Breast Cancer Aggressiveness. Clin Cancer Res 2019,25 (15), 4846-4858.

31. Wang, Y. C.; Wu, Y. S.; Hung, C. Y.; Wang, S. A.; Young, M. J.; Hsu, T. I.; Hung, J. J., USP24 induces IL-6 in tumor-associated microenvironment by stabilizing p300 and beta-TrCP and promotes cancer malignancy. Nat Commun 2018,9 (1), 3996.

32. Wang, Y. C.; Wang, S. A.; Chen, P. H.; Hsu, T. I.; Yang, W. B.; Chuang, Y. P.; Su, W. C.; Liaw, H. J.; Chang, W. C.; Hung, J. J., Variants of ubiquitin-specific peptidase 24 play a crucial role in lung cancer malignancy. Oncogene 2016,35 (28), 3669-80.

33. Luo, H.; Jing, B.; Xia, Y.; Zhang, Y.; Hu, M.; Cai, H.; Tong, Y.; Zhou, L.; Yang, L.; Yang, J.; Lei, H.; Xu, H.; Liu, C.; Wu, Y., WP1130 reveals USP24 as a novel target in T-cell acute lymphoblastic leukemia. Cancer Cell Int 2019,19, 56.

34. Peterson, L. F.; Sun, H.; Liu, Y.; Potu, H.; Kandarpa, M.; Ermann, M.; Courtney, S. M.; Young, M.; Showalter, H. D.; Sun, D.; Jakubowiak, A.; Malek, S. N.; Talpaz, M.; Donato, N. J., Targeting deubiquitinase activity with a novel small-molecule inhibitor as therapy for B-cell malignancies. Blood 2015, 125 (23), 3588-97.

\section{Figures}


Figure 1
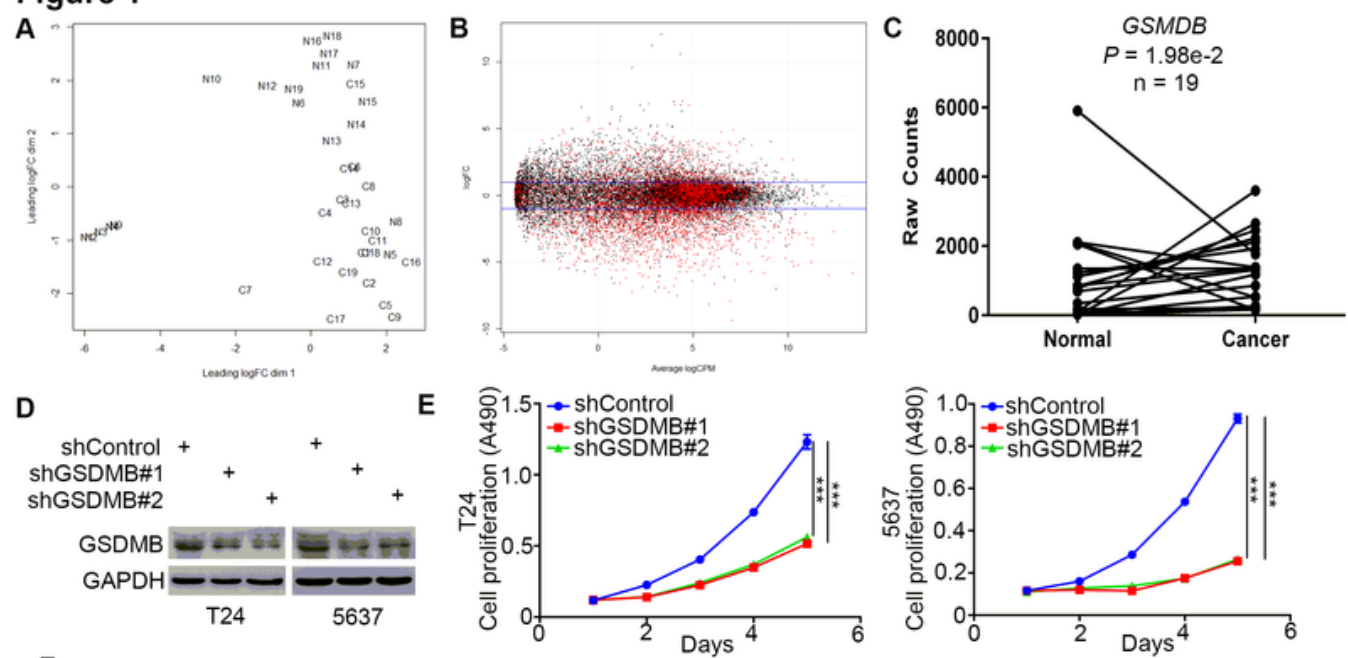

$\mathbf{F}$
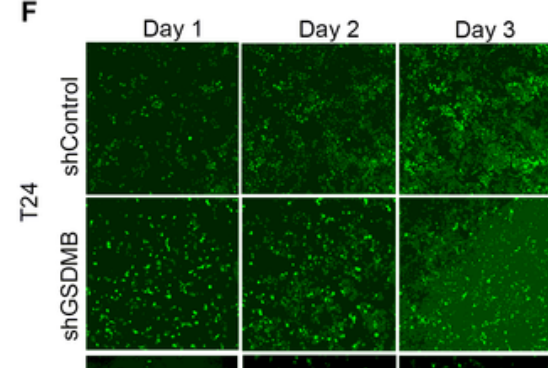

Day 4

Day 5
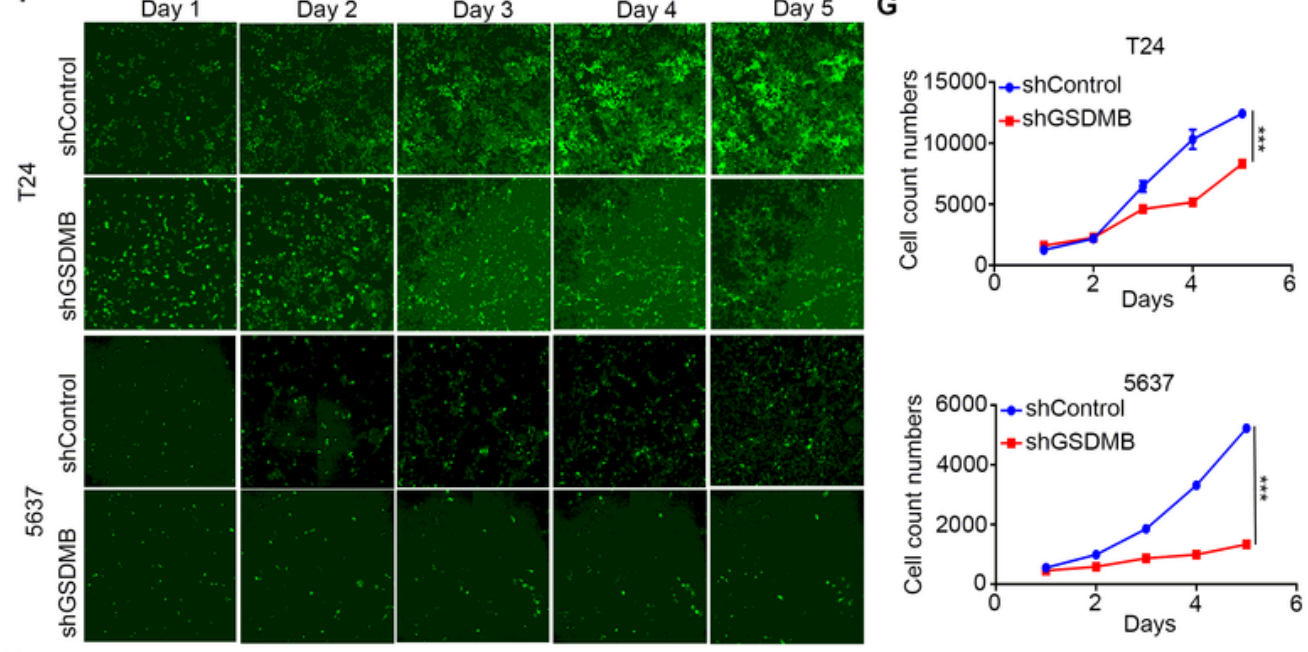

H
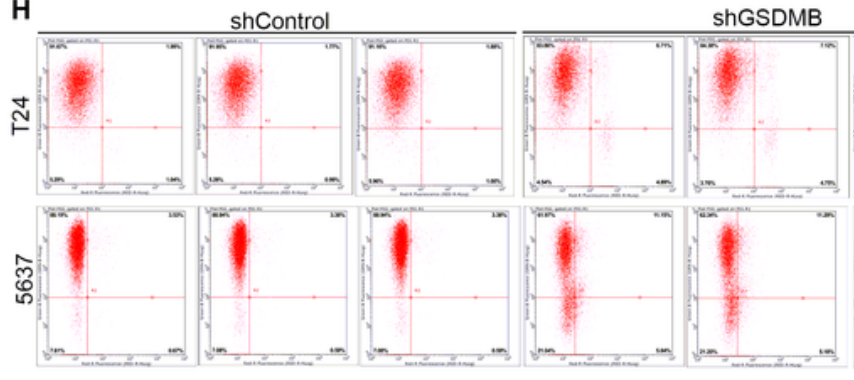

I
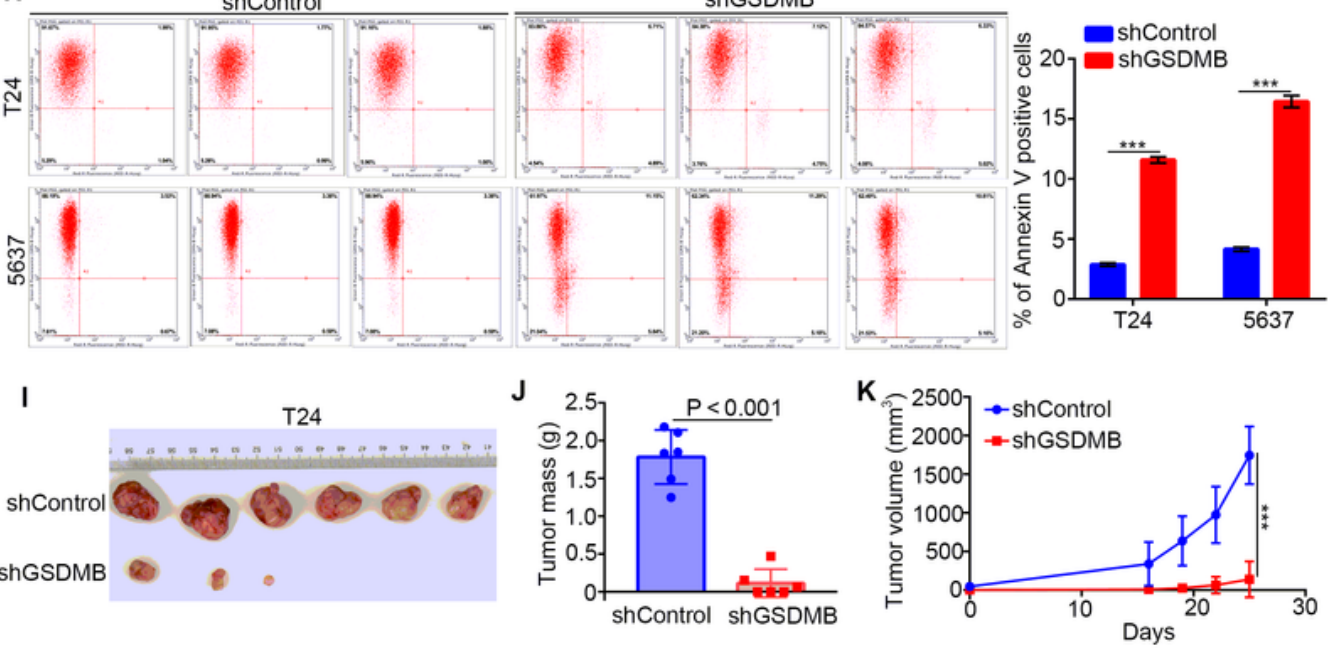

\section{Figure 1}

GSDMB promotes bladder cancer progression in vitro and in vivo. A-C. Analysis of RNA-Seq data from the TCGA dataset of bladder cancer containing 19 paired bladder cancer specimens and adjacent normal tissues. Panel A showed that normal and cancer tissues could be separated by dim2, which indicated that the data is stable for subsequent analysis. Panel $B$ is the general linear model to estimate whether there are differences among different groups of genes. The genes with $P$ value less than 0.05 are considered 
as differentially expressed genes in accordance with the zero hypothesis (the red dot in the figure). Panel $C$ indicated that GSDMB is upregulated in the above RNA-Seq dataset with $P=1.98 \mathrm{e}-2$. D-G. T24 and 5637 cell lines were infected with constructed plasmids. After infecting $72 \mathrm{~h}$, all cells were harvested for Western Blotting analysis (D), MTT assay (E) and celigo fluorescence cell count assay ( $F$ and G). All data were presented as Means $\pm S D(n=3)$. ${ }^{\star \star \star}, P<0.001$. H. T24 and 5637 cells were transfected with constructed plasmids for $72 \mathrm{~h}$. All cells were harvested and subjected to Annexin-V/APC assay. All data were showed as Means $\pm S D(n=3)$. ${ }^{\star \star \star}, P<0.001$. I-K. T24 cells were infected with constructed lentivirus to establish the stable knocking down cell lines. Then cells were injected subcutaneously into the nude mice to construct xenograft transplantation model. The image of xenografts was shown in (I), the tumor mass and volume were measured in $(J$ and $K)$. All data were presented as Means $\pm S D(n=6)$. ***, $P<$ 0.001 . 
Figure 2

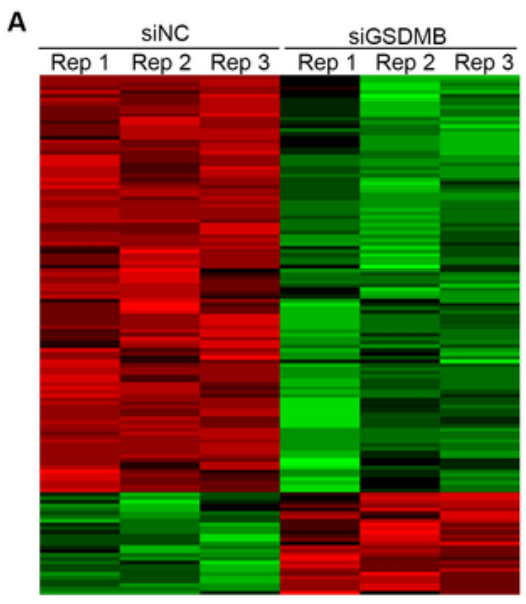

c

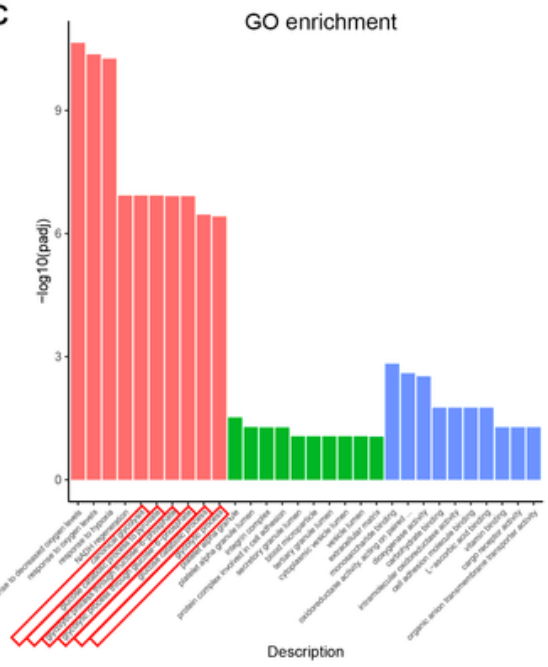

E
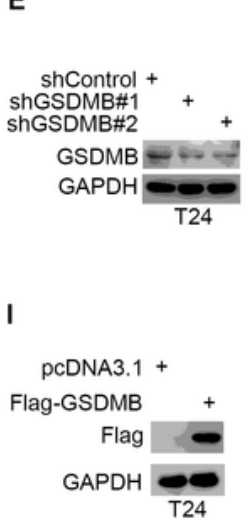

F
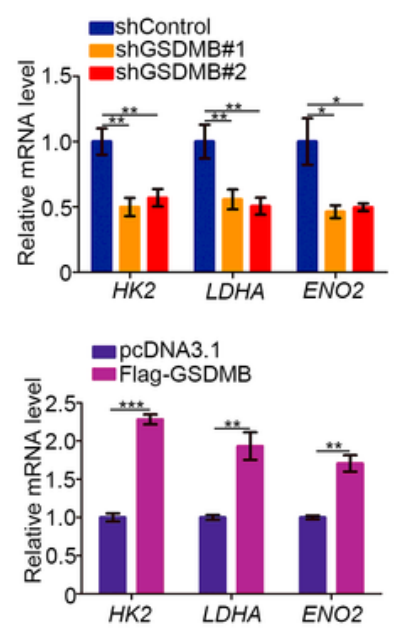

B

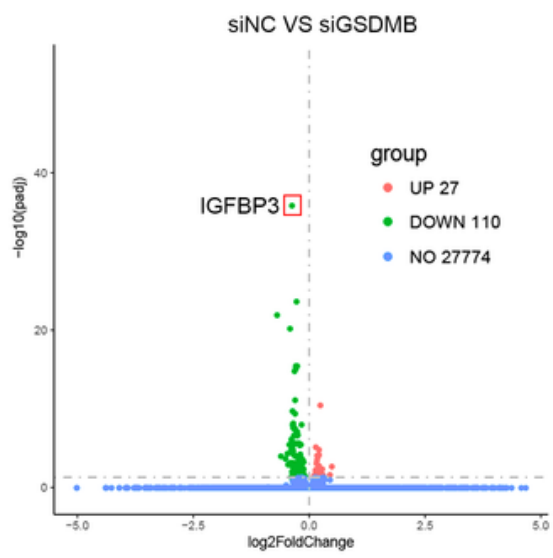

D

KEGG enrichment

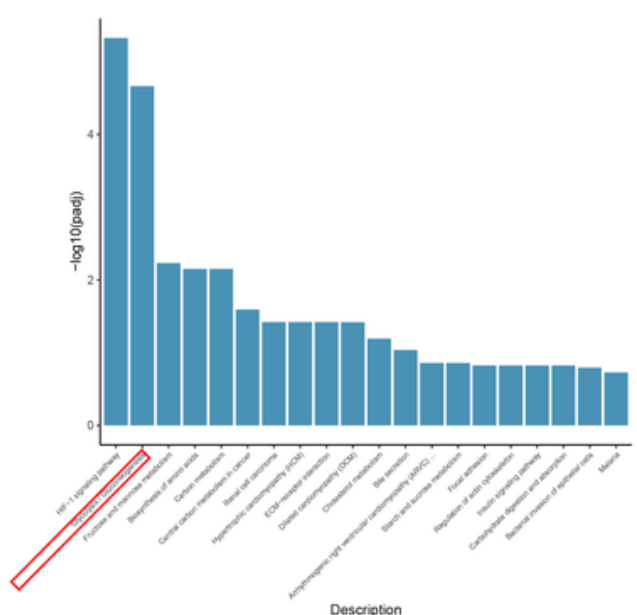

G

H

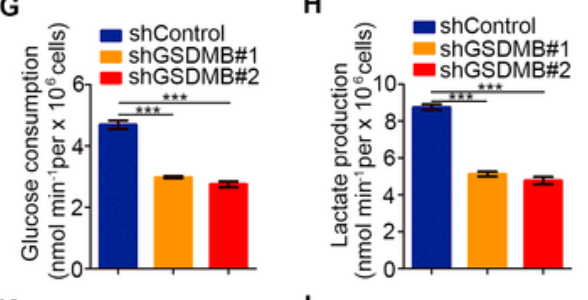

L

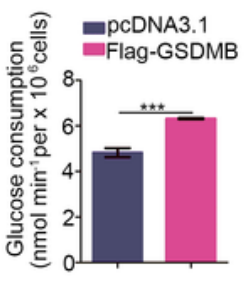

Figure 2

GSDMB regulates the glycolysis of bladder cancer cells. A-B. T24 cell lines were infected with constructed siGSDMB. After infecting 72h, cells were harvested for RNA-Seq. Heatmap (A) and volcano plot (B) were used to show the differential expressed genes. C-D. GO enrichment analysis (C) and KEGG enrichment analysis (D) based on differential genes through RNA-Seq. There were significant differences in glycolysis changes. E-H. T24 cell lines were infected with constructed plasmid. After infecting 48h and 
72h, all cells were harvested for RT-qPCR (F) and Western Blotting analysis (E). Glucose consumption (original level minus remaining amount) $(\mathrm{G})$ and lactate production $(\mathrm{H})$ were measured in the spent medium of T24 cells. All data were presented as Means $\pm S D(n=3)$. * $P<0.05$; **, $P<0.01$; ***, $P<$ 0.001 . I-L. T24 cell lines were infected with constructed plasmid. After infecting $48 \mathrm{~h}$ and $72 \mathrm{~h}$, all cells were harvested for RT-qPCR $(\mathrm{J})$ and Western Blotting analysis (I). Glucose consumption (original level minus remaining amount) $(\mathrm{K})$ and lactate production $(\mathrm{L})$ were measured in the spent medium of T24 cells. All data were presented as Means $\pm S D(n=3)$. **, $P<0.01 ; * \star *, P<0.001$.

\section{Figure 3}

A

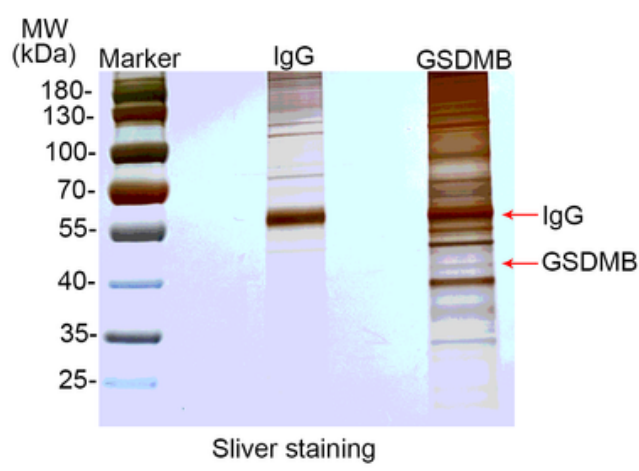

C

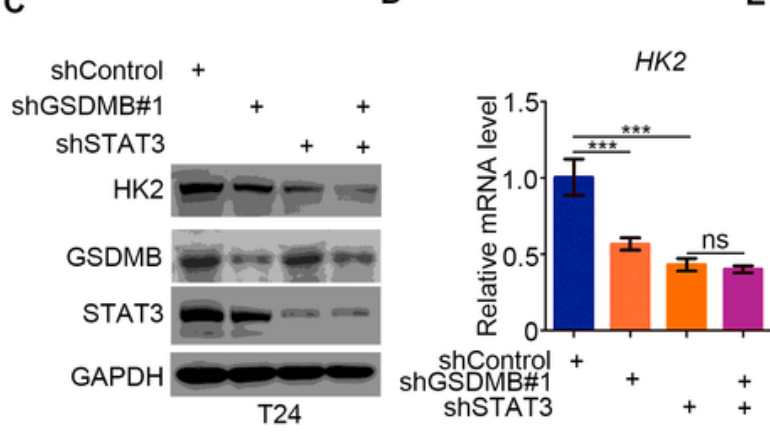

B

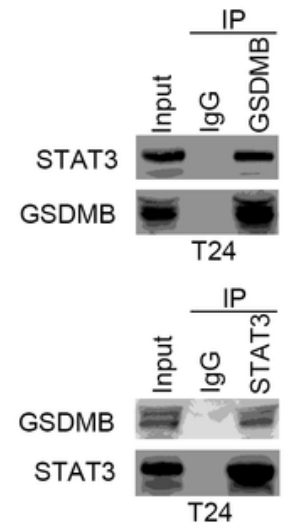

E

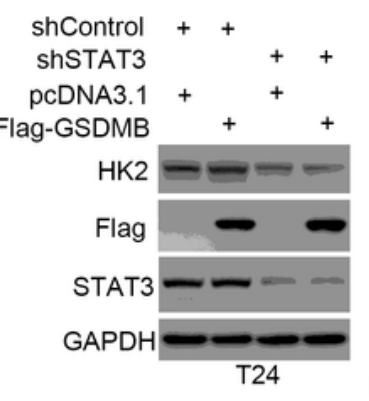

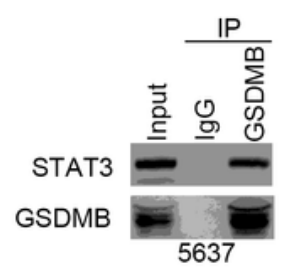

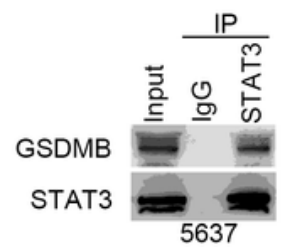

F

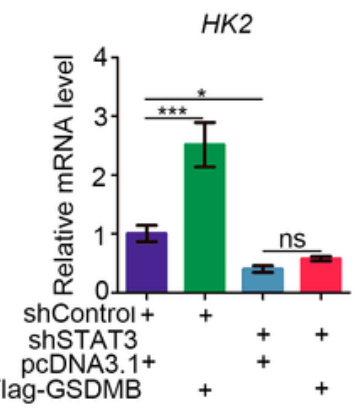

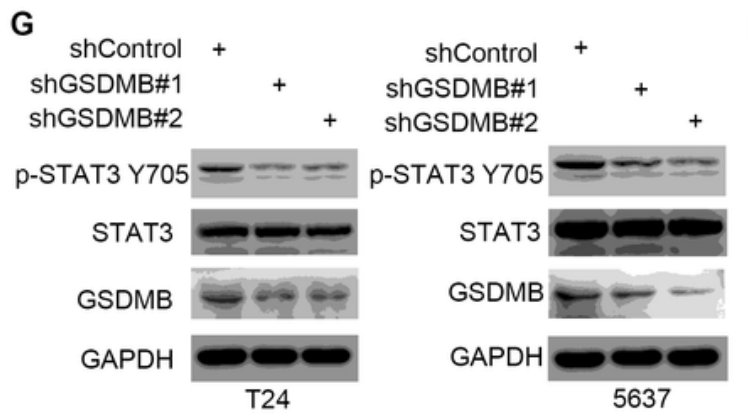

H
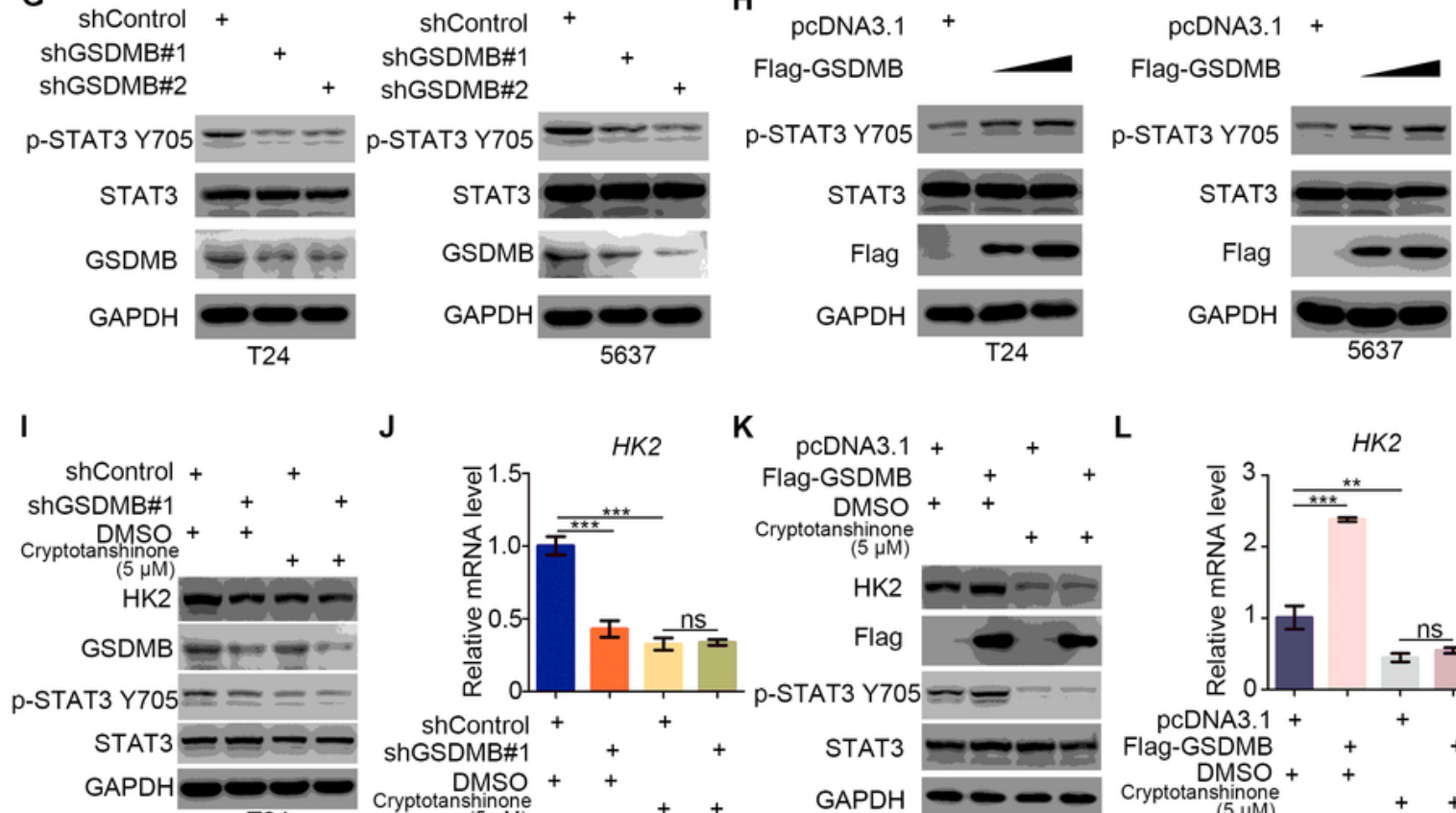

J
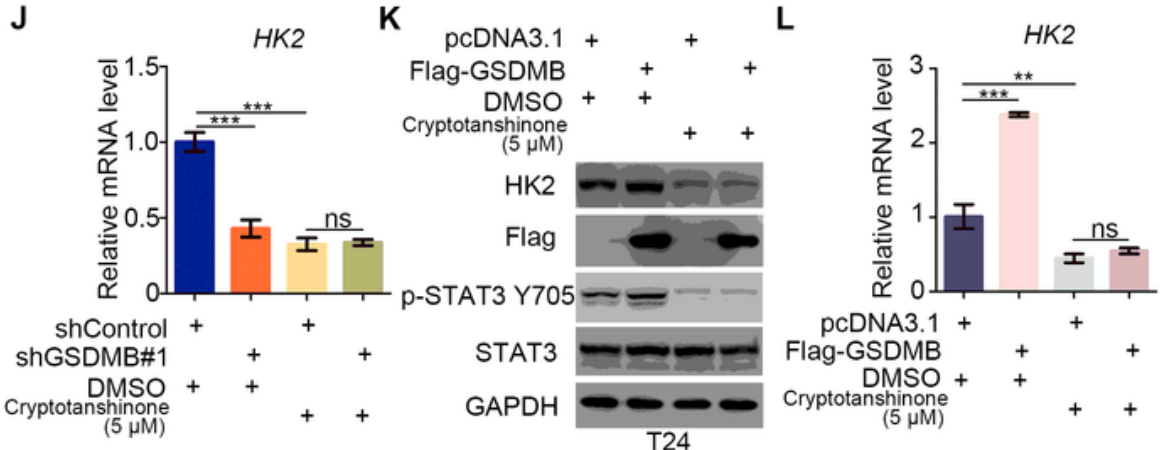


\section{Figure 3}

GSDMB regulates the glycolysis via activating STAT3 in bladder cancer cells. A. Gel electrophoresis was conducted after using GSDMB antibody for immunoprecipitation, then sliver staining was performed. The arrows indicated the protein at the expected molecular weight. B. Western blot analysis of coimmunoprecipitated protein samples from T24 and 5637 cells lines. G and H. T24 and 5637 cell lines were infected with indicated constructs (shGSDMB\#1, shGSDMB\#2 and Flag-GSDMB). After infecting 72h, all cells were harvested for Western Blotting analysis. C-F. T24 cell lines were infected with constructed plasmids (shGSDMB\#1, shSTAT3 and Flag-GSDMB). After infecting 48h and $72 \mathrm{~h}$, all cells were harvested for RT-qPCR (D and F) and Western Blotting analysis ( $C$ and $D)$. All data were presented as Means $\pm S D(n=3)$. * $P<0.05 ; * \star \star, P<0.001 ; n s$, no significant. I-L. T24 cell lines were infected with constructed plasmids (shGSDMB\#1 and Flag-GSDMB). After infecting 48h and 72h, the corresponding cell groups were treating with $5 \mathrm{uM}$ Cryptotanshinone for another $24 \mathrm{~h}$. Then all cells were harvested for RT-qPCR ( $\mathrm{J}$ and $\mathrm{L})$ and Western Blotting analysis (I and $\mathrm{K})$. All data were presented as Means $\pm \mathrm{SD}(\mathrm{n}=$ 3). **, $\mathrm{P}<0.01 ; * \star \star, \mathrm{P}<0.001 ; \mathrm{ns}$, no significant. 
Figure 4
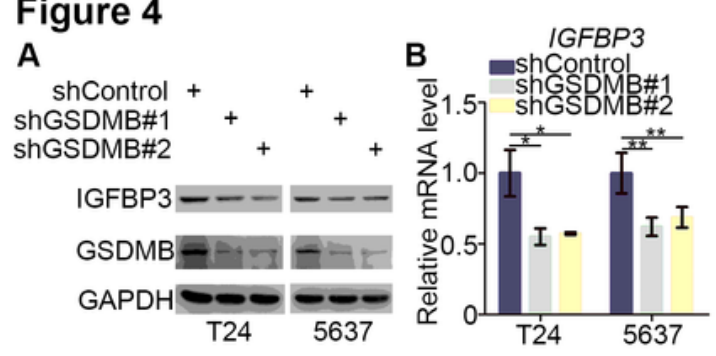

E
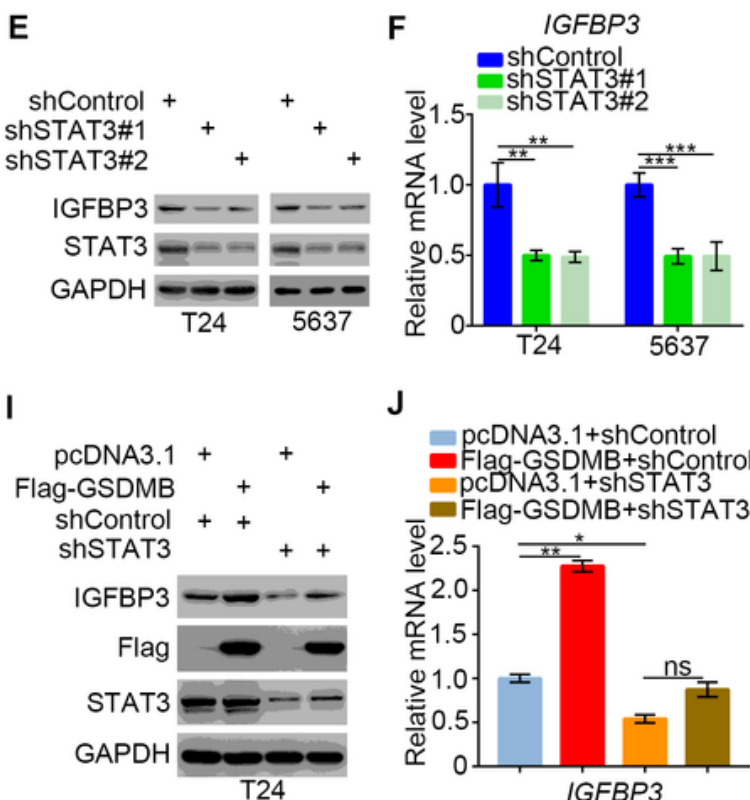

M

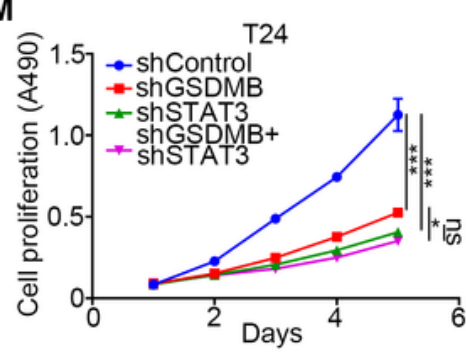

0
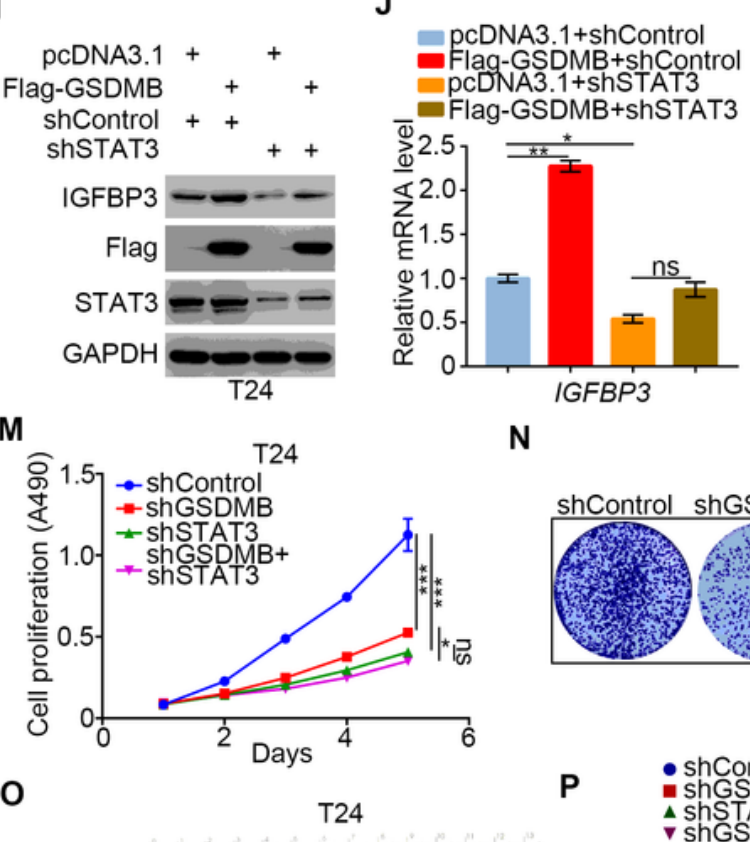

$\mathbf{N}$

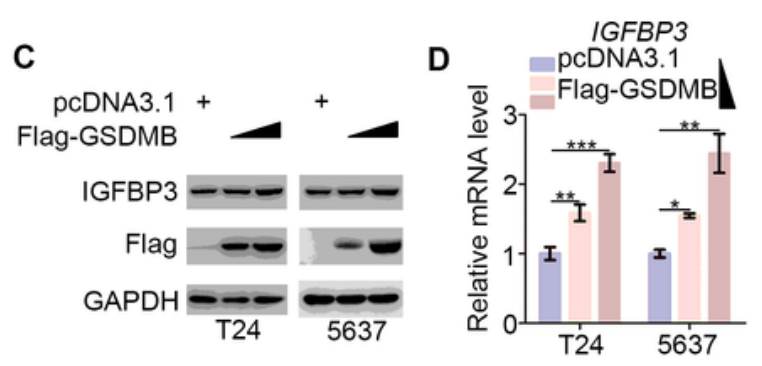

G

H

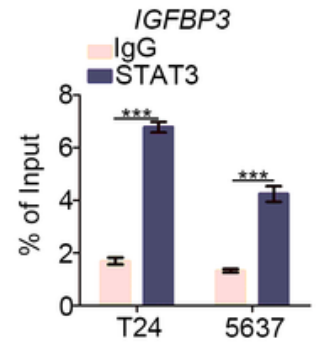

L
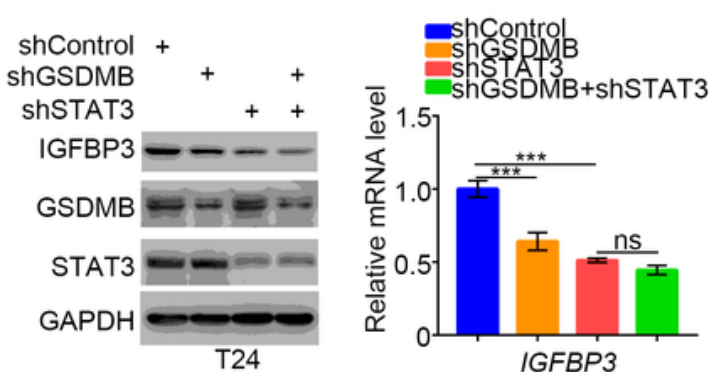

ChIP-qPCR

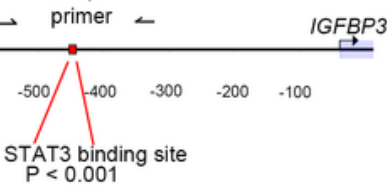

IGFBP3
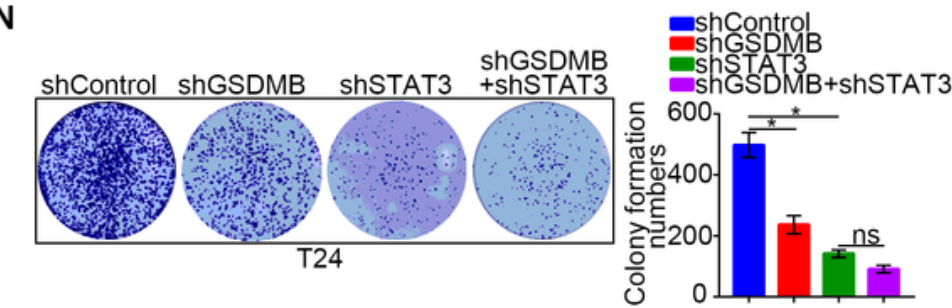

- shControl
- ShGSDMB
ShSTAT3

Q

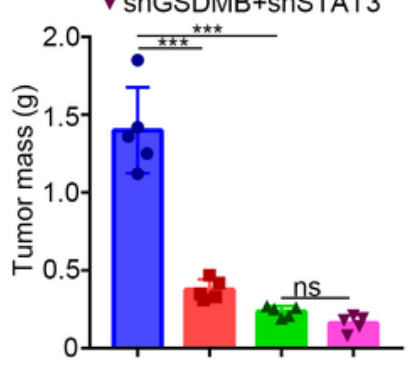

Figure 4

GSDMB-STAT3 signaling regulates IGFBP3 expression in bladder cancer. A-F. T24 and 5637 cell lines were infected with constructed plasmids (shGSDMB\#1, shGSDMB\#2, shSTAT3\#1, shSTAT3\#2 and FlagGSDMB). After infecting 48h and 72h, all cells were harvested for RT-qPCR (B, D, F) and Western Blotting analysis $(A, C, E)$. All data were showed as Means $\pm S D(n=3)$. * $P<0.05 ; * \star, P<0.01$; ***, $P<0.001$. G. The schematic diagram of STAT3 binding to the promoter position of IGFBP3. H. T24 and 5637 cell lines 
were treated according to the protocol of ChIP experiment, and the DNA samples were performed DNA agarose gel electrophoresis. All data were showed as Means $\pm S D(n=3)$. ${ }^{\star \star *}, P<0.001$. I-L. T24 cell lines were infected with constructed plasmids (shGSDMB, shSTAT3 and Flag-GSDMB). After infecting 48h and $72 \mathrm{~h}$, all cells were harvested for RT-qPCR ( $\mathrm{J}$ and $\mathrm{L}$ ) and Western Blotting analysis ( $\mathrm{l}$ and $\mathrm{K})$. All data were showed as Means \pm SD $(n=3) .{ }^{*}, P<0.05 ; * \star, P<0.01 ; * \star \star, P<0.001 ; n s$, no significant. M-N. T24 cell lines were infected with constructed plasmids (shGSDMB and shSTAT3). Cells were harvested for MTS assay $(M)$ and clone formation assay $(N)$. All data were showed as Means $\pm S D(n=3)$. *, $P<0.05$; ***, $P$ $<0.001$; ns, no significant. O-Q. T24 cells were infected with constructed lentivirus to establish the stable cell lines. Then cells were injected subcutaneously into the nude mice to construct xenograft transplantation model. The image of xenografts was shown in ( 0$)$, the tumor mass and volume were measured in $(P$ and $Q)$. All data were presented as Means $\pm S D(n=6) . * \star \star, P<0.001 ; n s$, no significant. 
A

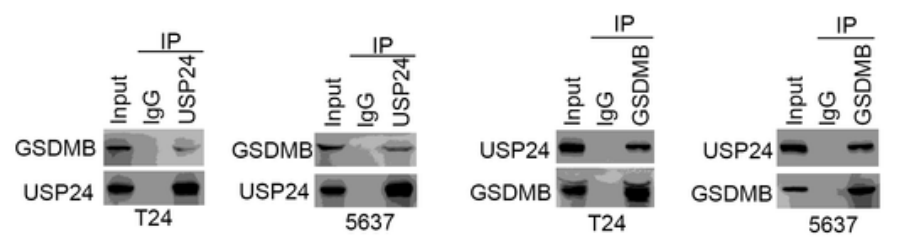

C
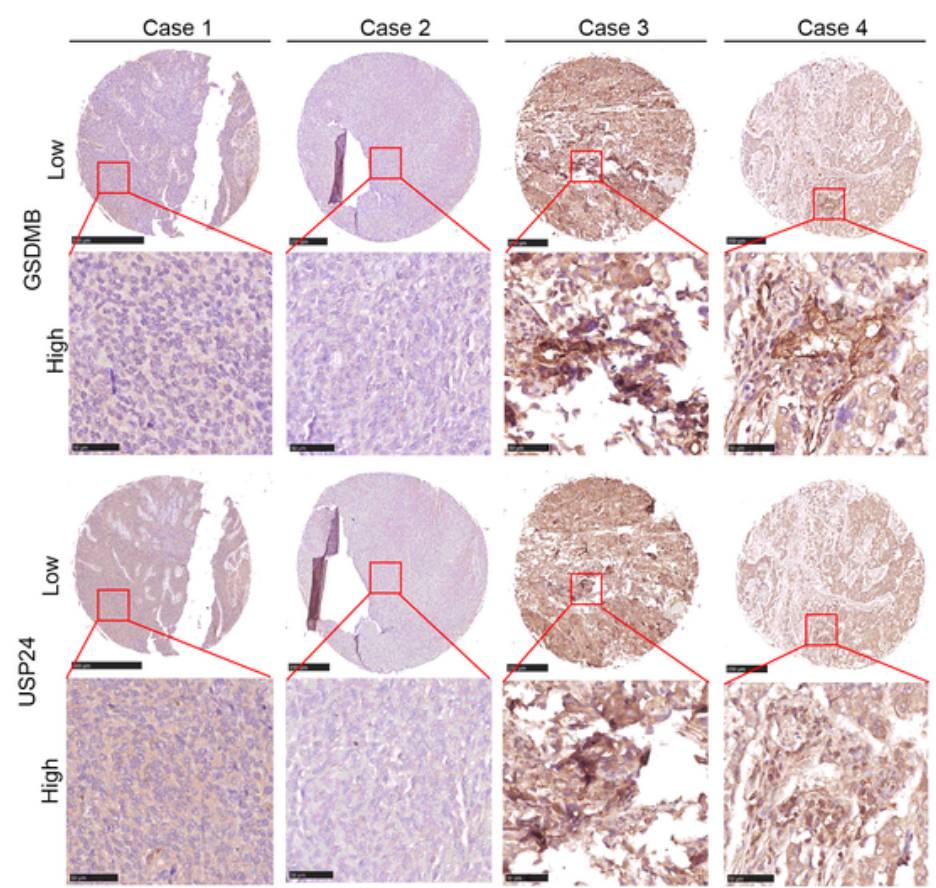

E
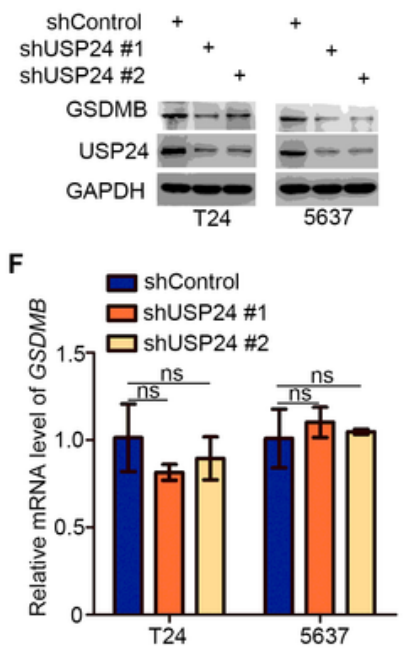

G

H
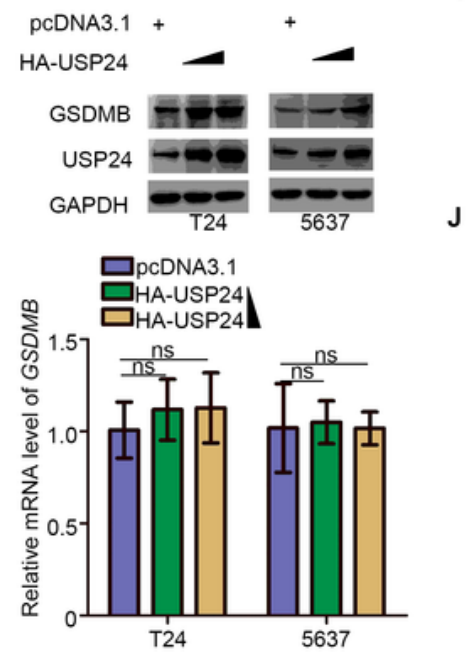

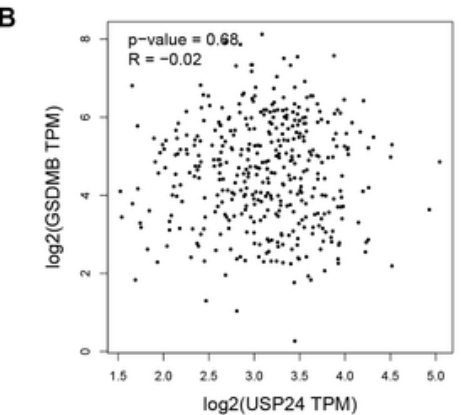

D
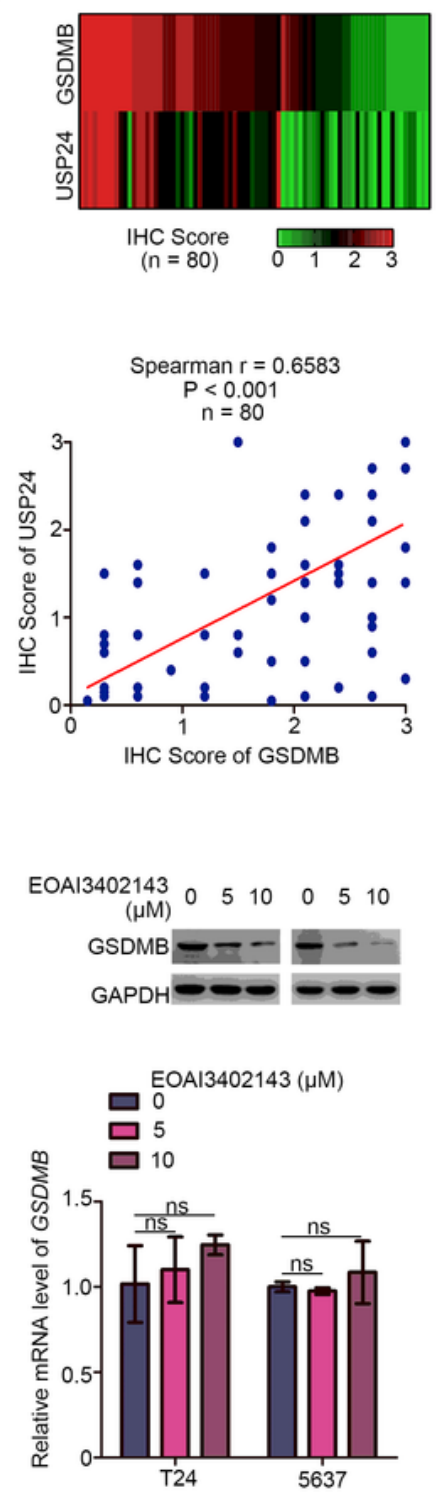

\section{Figure 5}

USP24 interacts with GSDMB to up-regulate the GSDMB protein level in bladder cancer. A. Western blot analysis of co-immunoprecipitated protein samples from T24 and 5637 cells lines. B. GEPIA network tool analyzed the RNA correlation between GSDMB and USP24. C. The typical IHC staining images using TMA tissue chip $(n=80)$. D. Heatmap and scatter diagram were used to reveal the correlation of protein expression between GSDMB and USP24 in bladder cancer patient specimens. ( $n=80$, spearman 
correlation $r=0.6583, \mathrm{P}<0.001)$. E-H. T24 and 5637 cell lines were infected with constructed plasmids (shUSP24\#1, shUSP24\#2, HA-USP24). After infecting 48h and 72h, all cells were harvested for RT-qPCR $(F$ and $H)$ and Western Blotting analysis $(E$ and $G)$. All data were showed as Means $\pm S D(n=3)$. ns, no significant. I-J. T24 and 5637 cell lines were treated with EOAl3402143 for $48 \mathrm{~h}$ and $72 \mathrm{~h}$. Then cells were harvested for RT-qPCR (I) and Western Blotting analysis $(J)$. All data were showed as Means $\pm S D(n=3)$. ns, no significant.

\section{Figure 6}

A

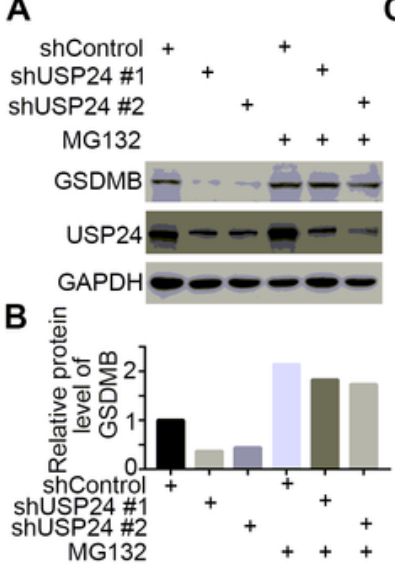

E

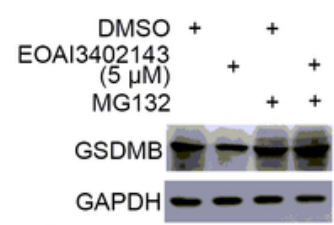

$\mathbf{F}$
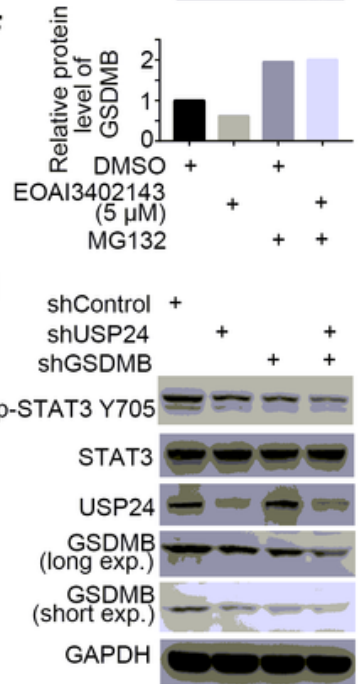

C ShControl shUSP24 shControl Times (h) $\frac{\text { + } 24816}{024816} \frac{\text { +HA-USP24 }}{024816}$

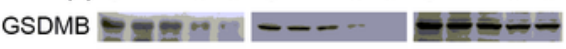
GAPDH

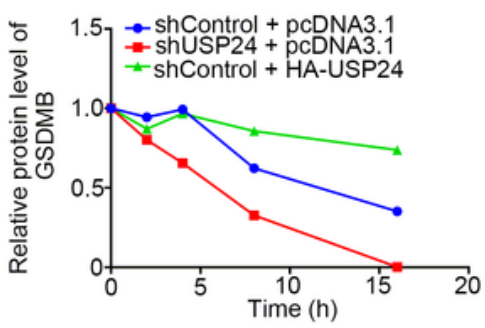

G

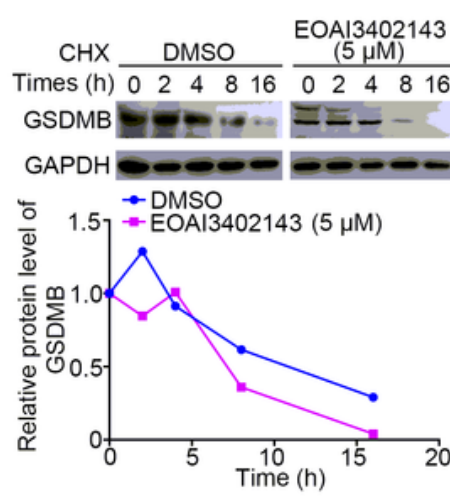

J

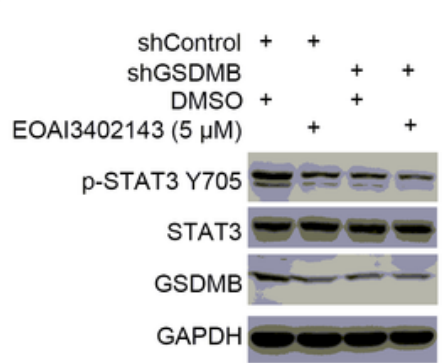

D

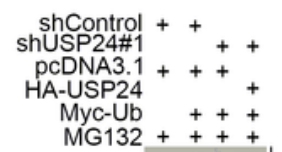

IB: Myc

IP: GSDMB

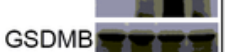

GSDMB $=---$

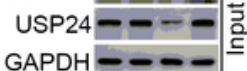

H

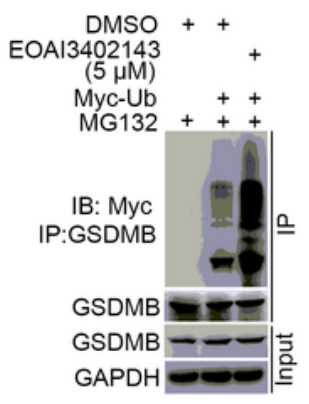

K

shControl ++++ ShGSDMB

pCDNA3.1 + + HA-USP24

p-STAT3 Y705 $==$

STAT3

USP24 - - - -

GSDMB $=$

GAPDH $--\infty$

L

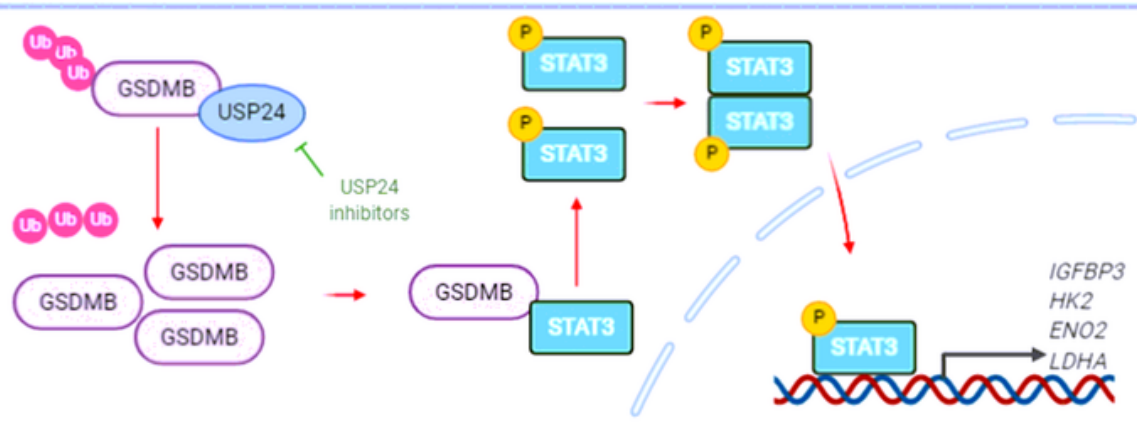

Figure 6 
USP24 stabilizes GSDMB to promote STAT3 phosphorylation in bladder cancer cells. A-B. T24 and 5637 cell lines were infected with constructed plasmids (shUSP24\#1, shUSP24\#2). After infecting 48h and $72 \mathrm{~h}$, the corresponding groups were treated with MG132 for another $12 \mathrm{~h}$. All cells were harvested for Western Blotting analysis. C. T24 cells were infected with indicated plasmids (shUSP24 and HA-USP24). After infecting $72 \mathrm{~h}$, cells were treated with Cycloheximide (CHX) and all cells were collected for Western Blotting analysis at different time points. D. T24 cells were infected with indicated plasmids (shUSP24\#1, HA-USP24 and Myc-Ub). After 24h, cells were treated with MG132 for 12h. Then all cells were collected for Western Blotting analysis. E-F. T24 cells were treated with 5uM EOAl3402143. After 48h, cell were treated with MG132 for another 24h. Then all cells were collected for Western Blotting analysis. G. T24 cells were treated with 5uM EOAl3402143. After 72h, cells were treated with Cycloheximide (CHX) and all cells were collected for Western Blotting analysis at different time points. H. T24 cells were treated with 5uM EOAI3402143. After 48h, T24 cells were infected with indicated plasmids (Myc-Ub) for $24 \mathrm{~h}$. Then cells were treated with MG132 for $12 \mathrm{~h}$. All cells were collected for Western Blotting analysis. I-K. T24 cell lines were infected with constructed plasmids (shUSP24, shGSDMB, HA-USP24). Cells were treated with 5 uM EOAl3402143 in $(\mathrm{J})$. After infecting or treating 72h, all cells were harvested for Western Blotting analysis. L. The schematic diagram for USP24 stabilizing GSDMB to promote STAT3 phosphorylation, which subsequently increased the expression of glucose metabolism-related genes (e.g., HK2, LDHA, ENO2, and IGFBP3) and promoted bladder cancer cell proliferation.

\section{Supplementary Files}

This is a list of supplementary files associated with this preprint. Click to download.

- Supplementaryinformation.docx

- Tables4RNASeqofGSDMB.xlsx

- TableS5MassspectrometryofGSDMB.xIsx 